\title{
Flight Dynamics Analyses of a Propeller-Driven Airplane (II): Building a High-Fidelity Mathematical Model and Applications
}

\author{
Chang-Joo Kim*, Sang Ho Kim**, TaeSan Park***, Soo Hyung Park**** and Jae Woo Lee***** \\ Department of Aerospace and Information Engineering, Konkuk University, Seoul 143-701, Korea
}

\section{Joon Soo Ko K***** $^{* * 2}$}

School of Aerospace and Mechanical Engineering, Korea Aeronautical University, Seoul 143-701, Korea

\begin{abstract}
This paper is the second in a series and aims to build a high-fidelity mathematical model for a propeller-driven airplane using the propeller's aerodynamics and inertial models, as developed in the first paper. It focuses on aerodynamic models for the fuselage, the main wing, and the stabilizers under the influence of the wake trailed from the propeller. For this, application of the vortex lattice method is proposed to reflect the propeller's wake effect on those aerodynamic surfaces. By considering the maneuvering flight states and the flow field generated by the propeller wake, the induced velocity at any point on the aerodynamic surfaces can be computed for general flight conditions. Thus, strip theory is well suited to predict the distribution of air loads over wing components and the viscous flow effect can be duly considered using the 2D aerodynamic coefficients for the airfoils used in each wing. These approaches are implemented in building a high-fidelity mathematical model for a propeller-driven airplane. Flight dynamic analysis modules for the trim, linearization, and simulation analyses were developed using the proposed techniques. The flight test results for a series of maneuvering flights with a scaled model were used for comparison with those obtained using the flight dynamics analysis modules to validate the usefulness of the present approaches. The resulting good correlations between the two data sets demonstrate that the flight characteristics of the propeller-driven airplane can be analyzed effectively through the integrated framework with the propeller and airframe aerodynamic models proposed in this study.
\end{abstract}

Key words: propeller-driven airplane, flight dynamics modeling, vortex lattice method, blade element method, strip theory

\section{Introduction}

A high-fidelity mathematical model for flight dynamics analyses of a propeller-driven airplane requires a detailed model of the propeller's aerodynamics and inertial loads. Also, the propeller wake effect should be duly considered to predict accurately the aerodynamic loads generated by the airframe. For these purposes, the first paper in this series focused on building a propeller model and the major approaches related to this paper were briefly introduced as far as required. This paper focuses on detailed aerodynamic models of the fuselage and wing components for their integration with the propeller model developed in the previous paper. The relative air velocities on the airframe aerodynamic surfaces are affected by the propeller wake as well as the air velocity induced by their wing loadings. Also, unsteady flight conditions should be considered for an accurate flight dynamics analysis. The vortex lattice method (VLM) $[1,2]$ can be an effective alternative in handling such a complex flow environment. The VLM can compute the lift, induced drag, and pitching moment with good accuracy but it fails to consider the viscous flow effect because of its basis
This is an Open Access article distributed under the terms of the Creative Commons Attribution Non-Commercial License (http://creativecommons.org/licenses/by$\mathrm{nc} / 3.0 /$ which permits unrestricted non-commercial use, distribution, and reproduction in any medium, provided the original work is properly cited.

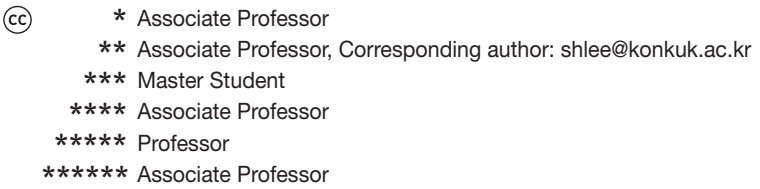


in potential flow theory. To address this limitation, strip theory [3] is used in this paper to accurately compute the non-linear aerodynamic forces and moments over the wing using the induced velocity field predicted with the VLM. This integrated approach with both the VLM and strip theory can provide accurate computations of aerodynamic forces and moments for the propeller-driven airplane because its wing components are generally characterized by a high aspect ratio.

The induced velocities generated by the propeller and wings depend greatly on the strengths of the vortex filaments. Also, the wing loadings, which are affected by the induced velocities, determine the circulations (the strengths of the bound vortices). Thus, a recursive approach is required to compute the induced velocity fields under given flight conditions. Because such an iterative procedure takes much computing time to analyze the propeller's wake and applying the VLM, its implementation should be planned carefully to build an efficient mathematical model for flight dynamics analyses. For this purpose, a series of trade-off studies was performed first to determine the minimum numbers of blade elements and vortex lattices without degrading the prediction accuracy. Second, the computational procedures were designed carefully for the efficient integration of all aerodynamic models to save computing time. Detailed computational procedures and aerodynamic modeling techniques, including use of a wind tunnel database for the airframe, are introduced in the following sections. The results of this study are applied to the flight dynamics analysis of the KLA-100 airplane to prove the utility of the present integrated math model.

\section{Airframe aerodynamic models}

\subsection{Vortex lattice method (VLM)}

Aerodynamic models for the fuselage and wings were developed for an airplane with a front-installed propeller. With such an installation, the effect of the airframe air loads on the propeller aerodynamics can be ignored with little degradation in model fidelity. Fig. 1 shows a schematic vortex lattice layout for the present study. The 24 vortex lattices were used to describe the aerodynamic shape of the wing and the control surface. These were modeled with an equal number of line-vortex filaments that have their leading edges at the quarter chord of each lattice. They are stretched downstream and can be described by horseshoetype vortices. The strengths of all vortex filaments can be determined by applying flow tangency conditions at the control point located at the three-quarter chord of each vortex lattice. The relative air velocity at the $j^{\text {th }}$ control point can be written as

$$
\mathbf{V}_{j}=\mathbf{V}_{\infty}+\boldsymbol{\omega} \times \mathbf{r}_{j}+\mathbf{V}_{i, j}
$$

where $\mathbf{r}_{j}$ and $\mathbf{V}_{i, j}$ represent the position vector measured from the airplane's center of gravity (CG) and the induced velocity, respectively. The vectors, $\mathbf{V}_{\infty}$ and $\boldsymbol{\omega}$, denote the linear and angular velocities of the airplane.

The induced velocity $\mathbf{V}_{i, j}$ can be obtained by applying the Biot-Savart law for all line vortex elements of the propeller and wings. A viscous core model is used to avoid singular behavior when a line-vortex element approaches the control point [4]. The induced velocity $\mathbf{V}_{j}$ at the $\mathrm{j}^{\text {th }}$ control point can be derived by summing all contributions from each vortex filament, as shown in Eq. (2), when the total number of line-vortex elements for the propeller, fuselage, and wing components is $K$.

$$
\mathbf{V}_{j}=\mathbf{V}_{\infty}+\boldsymbol{\omega} \times \mathbf{r}_{j}+\sum_{k=1}^{K} \mathbf{I}_{j, k} \Gamma_{k}
$$

The vortex strengths $\Gamma_{k}(k=1 . \ldots, K)$ can be determined

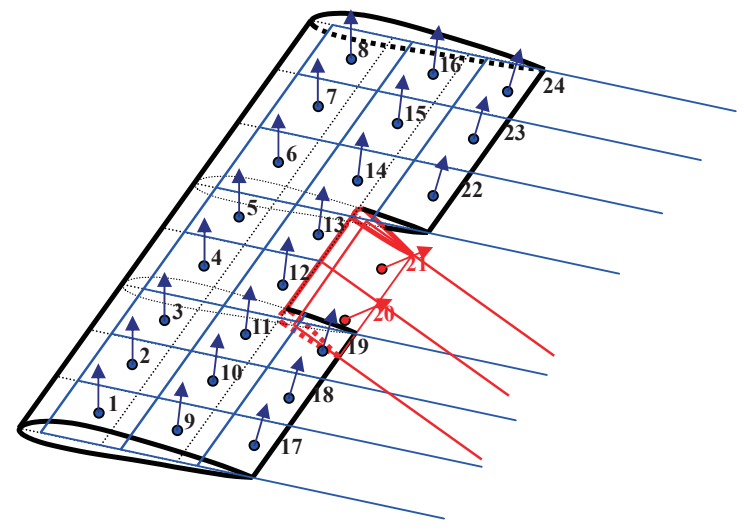

Fig. 1. Schematic geometry of vortex lattices for a wing with a control surface

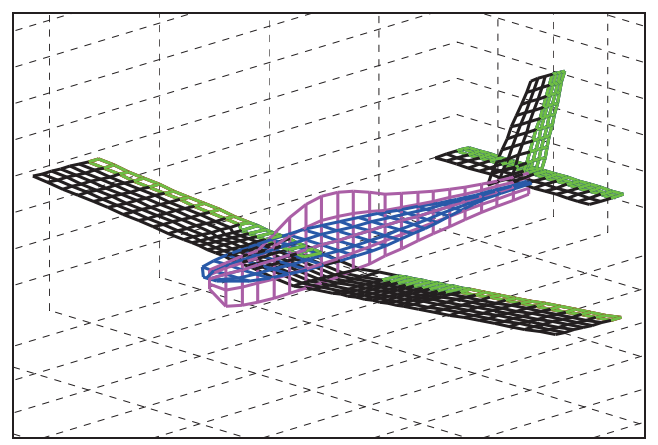

Fig. 2. Vortex lattice distributed over the aerodynamic surfaces for the VLM 
by imposing the following flow tangency conditions and by solving the resulting system of linear algebraic equations. Fig. 2 shows a schematic of the vortex lattice geometry used for this study. The fuselage was modeled using the horizontal and vertical vortex lattices to reflect the effects of different flight directions. The geometry of the vortex filaments for the main wing, with the aileron and the flap, is shown in Fig. 3. The maximum wake age of the wing trailed wakes is established to cover their effect on the empennage.

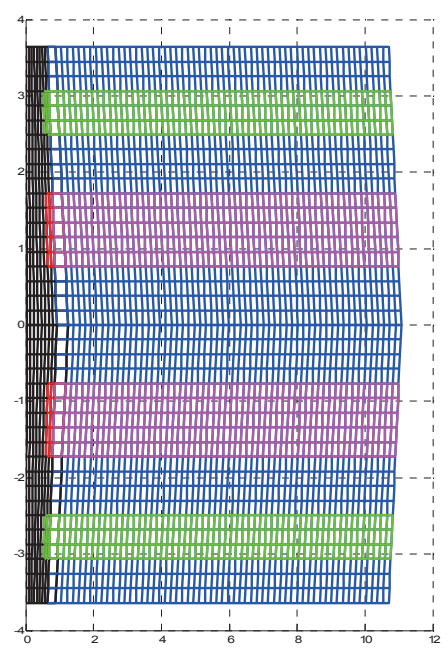

Fig. 3. Vortex filament wake model for main wing with control surfaces
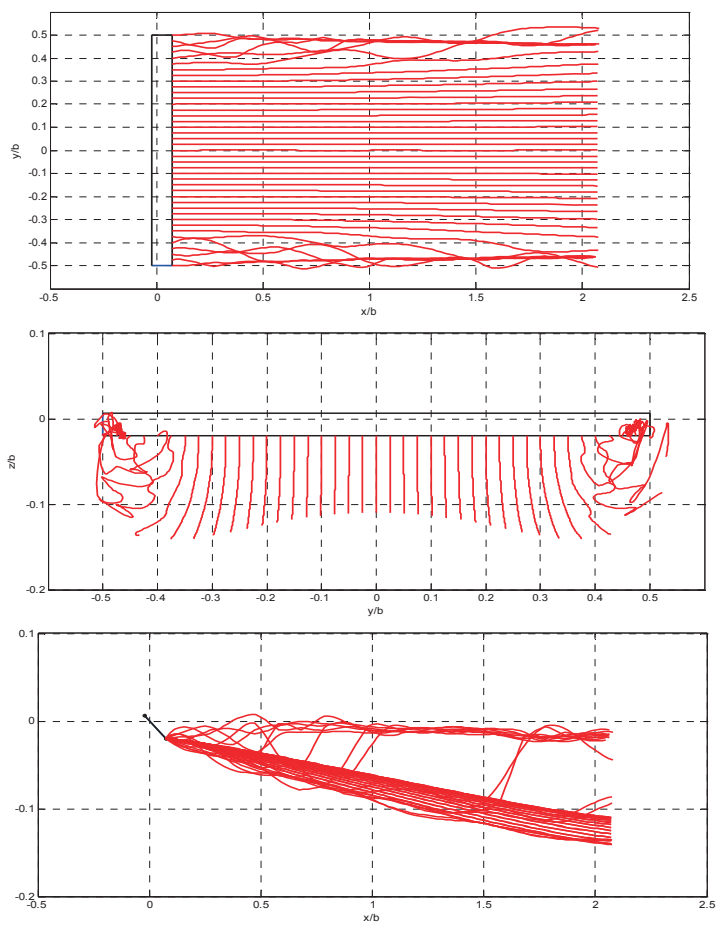

Fig. 4. Predicted geometry of the trailed vortex filament for the wing (aspect ratio $=10^{\circ}$ and angle of attach $=15^{\circ}$ )
An accurate computation of the induced velocity can be obtained with a more realistic wake geometry using free-wake theory [4]. As an example of its application, Fig. 4 shows free-wake geometry predicted using the methods in Ref. [5] around a wing with an aspect ratio of 10 at the angle of attack (AoA) of $15^{\circ}$. This shows that the tip vortex is established through the rollup process among the trailed wakes around the wing tip. With such realistic geometry of the wake structure, more accurate predictions of wing air loads may be possible. However, the computation of freewake geometry is extremely expensive, while the air loads predicted with free-wake geometry presents nearly the same level of accuracy as those with the prescribed wake in the present applications of the propeller-driven airplane.

\subsection{Strip theory}

The aerodynamic loads over a wing component were computed using strip theory. For this purpose, the aerodynamic coefficients of wing airfoils were computed and tabulated with varying AoA using the Navier-Stokes solver KFLOW, developed by one of the authors [6]. Fig. 5 shows the striped wing elements for the main wing and the empennage to compute the sectional lift, drag, and pitching moment. Once the induced velocity at the aerodynamic center of each wing element is available, the aerodynamic forces and moments generated by a striped wing component with the effective AoA $\alpha_{j}$ and the spanwise width $d s_{j}$ can be represented for the sectional lift by
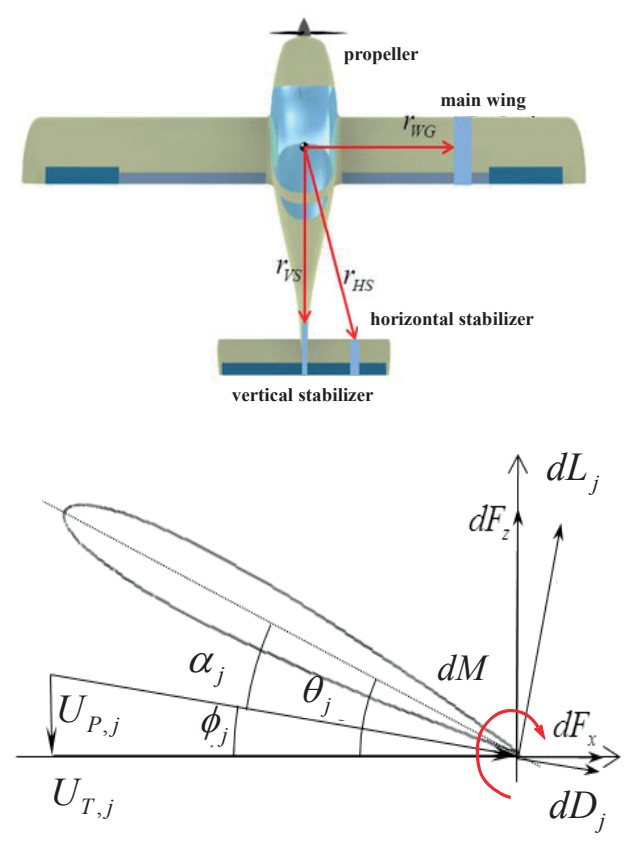

Fig. 5. Aerodynamic forces over a striped wing element for the main and tail wings 


$$
d L_{j}=\frac{1}{2} C_{L}\left(\alpha_{j}\right) \rho V_{j}^{2} c_{j} d s_{j}
$$

The tangential and perpendicular velocity components $\left(U_{T, j}, U_{P, j}\right)$ are readily defined using Fig. 5 . The effective AoA $\alpha_{j}=\theta_{j^{-}} \phi_{j}$ and the resulting flow velocity $V_{j}=\sqrt{U_{T, j}^{2}+U_{P, j}^{2}}$ can be obtained with reference to the same figure. Thus, the aerodynamic forces $d \mathbf{f}_{j}^{(a e o)}$ and moments $d \mathbf{m}_{j}^{(a e o)}$ generated by the striped wing element can be represented as shown in Eqs. (4) and (5). Their contribution to total aerodynamic loads around the airplane's CG can be easily formulated by considering the coordinate transformation relations and the relative position vector from the CG to the aerodynamic center of the wing element.

$$
\begin{aligned}
& d \mathbf{f}_{j}^{(a e o)}=\left(d L_{j} \sin \phi_{j}+d D_{j} \cos \phi_{j}\right) \mathbf{u}_{x, j}+\left(d L_{j} \cos \phi_{j}-d D_{j} \sin \phi_{j}\right) \mathbf{u}_{n, j} \\
& d \mathbf{m}_{j}^{(a e o)}=d M_{j} \mathbf{u}_{s, j}
\end{aligned}
$$

where $\mathbf{u}_{x, j}$ and $\mathbf{u}_{s, j}$ represent unit vectors in the directions of the airfoil chord and wing span, respectively and $\mathbf{u}_{n, j}=\mathbf{u}_{x, j} \times \mathbf{u}_{s, j}$. The formulae given in Eqs (4) and (5) can be applied for all wing components, such as the main wing and the horizontal and vertical stabilizers. The changes in aerodynamic loads for a striped wing element due to the installed control surface deflection are computed using the aerodynamic coefficients, which are tabulated using the 2D Navier-Stokes analyses of the airfoils with a control surface with variations of control surface deflection angle using KFLOW. The aerodynamic coefficients to reflect the effects of the angular deflections of each control surface are tabulated for 10 different angles of attack of the relevant wing (ranging from -10 to $20^{\circ}$ ). Those were computed for five deflection angles for both the flap and the aileron ( -20 to $\left.20^{\circ}\right)$ and for seven angular positions of the rudder $\left(-30\right.$ to $\left.30^{\circ}\right)$.

\subsection{Aerodynamic models for the fuselage and con- trol surfaces}

In this study, the air loads due to the fuselage were computed using a wind tunnel database. Wind tunnel tests were performed using the scaled airframe model with all wing components installed for the KLA-100 airplane without the propeller. The static fuselage aerodynamic coefficients were tabulated by subtracting the wing contributions predicted using the strip theory from the wind tunnel data
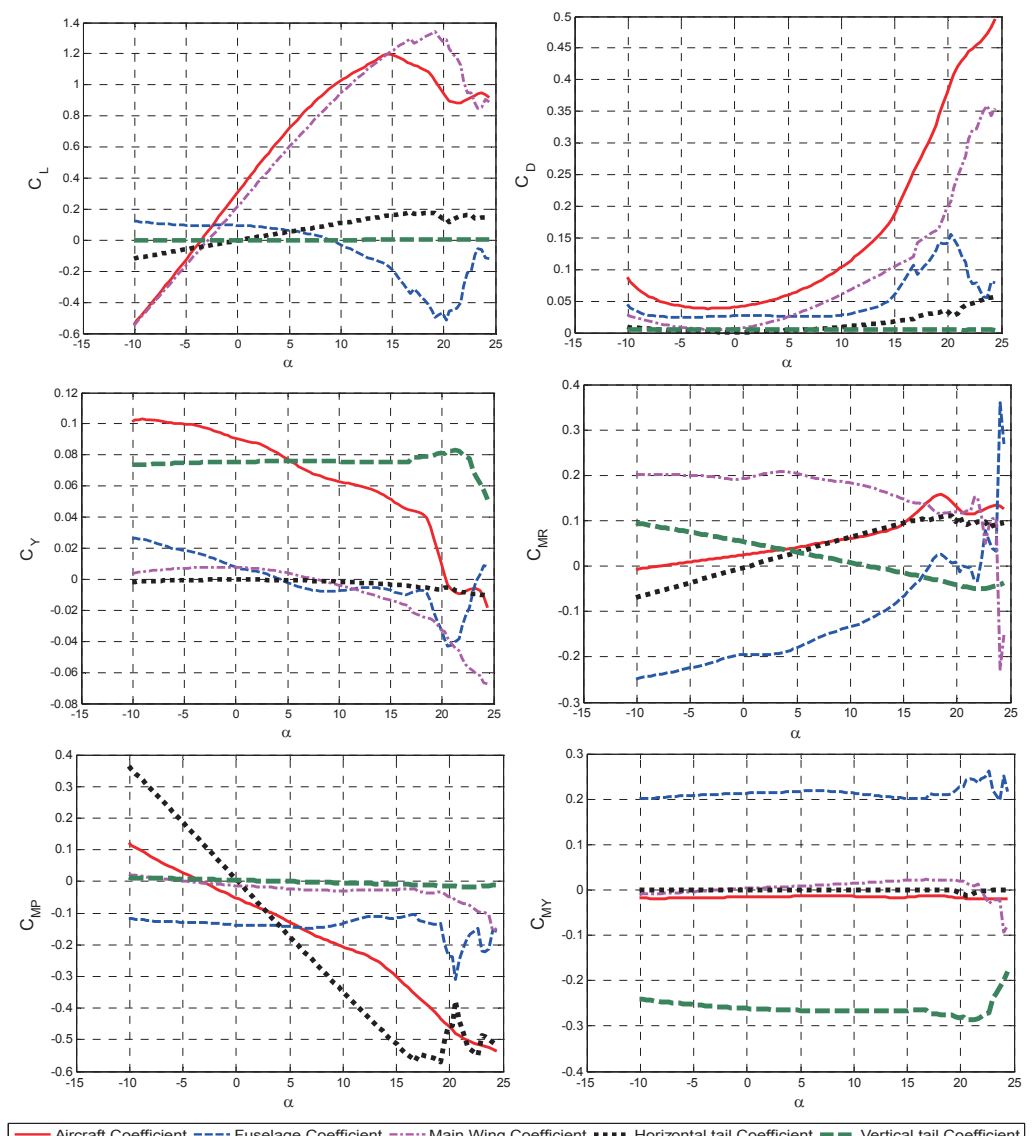

Fig. 6. Aerodynamic coefficients for each modeling elements at zero sideslip angle 
for the airframe. Thus, the airframe aerodynamic coefficients predicted with the present methods are the same as those measured through a series of wind tunnel tests when the propeller wake effects are ignored. Fig. 6 shows the aerodynamic coefficients for each modeling element when the sideslip angle is zero. The coefficients for the airframe are the wind tunnel test data for the aircraft and those for the fuselage are the computed results using the method mentioned above.

The fuselage air loads due to rotational motions of the airplane and the propeller wakes can be considered in principle by isolating the related delta-air loads from the static. However, this kind of modeling can add extreme complexity in its implementation while minor improvements
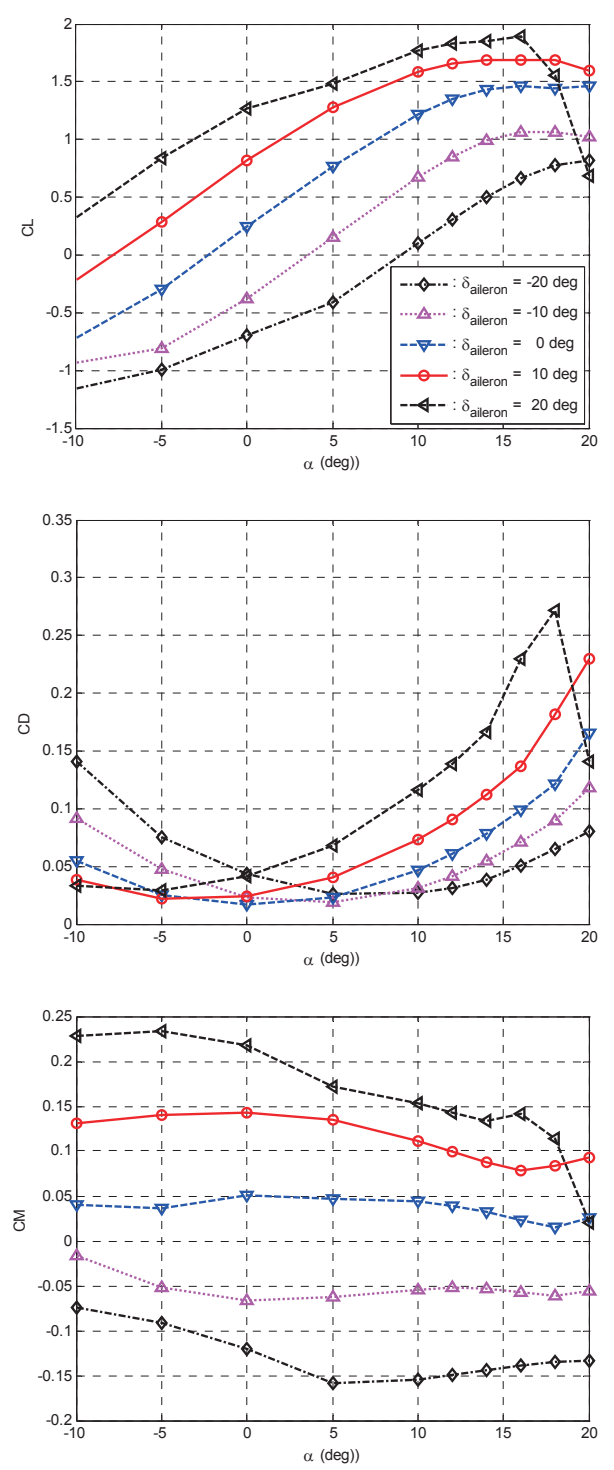

Fig. 7. Airfoil aerodynamic coefficients with varying control surface deflections are expected in prediction accuracy. In a case when aerodynamic surfaces, such as the dorsal or ventral fins are installed, the propeller wake effects on those surfaces can be estimated readily from air load computations with their VLM models. The aerodynamic coefficients for an airfoil with control surface deflection are computed using the NavierStokes equation solver and tabulated to consider the control effectiveness. In the range of the airfoil AoA from -20 to $20^{\circ}$, five deflection angles of the control surfaces were selected to create the aerodynamic tables for the lift, drag, and pitching moment coefficients. Fig. 7 shows an example variation of the aerodynamic coefficients due to the aileron deflection angle.

\section{Flight dynamics model}

The rigid-body motions for the airplane can be obtained considering the forces and moments generated by all modeling components. As an example, the lateral angular motion equation is represented by

$$
\begin{aligned}
& I_{y y} \dot{q}-I_{x y} \dot{p}-I_{y z} \dot{r}+\left(I_{x x}-I_{z z}\right) p r+I_{z x}\left(p^{2}-r^{2}\right)+I_{y z} p q-I_{y x} q r \\
& =M_{P}+M_{W}+M_{F}+M_{V}+M_{H}
\end{aligned}
$$

The subscripts $P, W, F, V$ and $H$ represent the contributions from the propeller, main wing, fuselage, horizontal, and vertical stabilizers, respectively. The kinematic relationships for the attitude rates and the airplane position at the local horizontal reference frame are added in the motion equations to obtain the linear and angular position of the airplane in three-dimensional space. The aircraft motion equations are inherently implicit because they have linear and angular acceleration terms in the right-hand side through the propeller inertial forces and moments. An explicit form of the motion equations is derived for convenience considering the mass properties of the propeller, as explained in Ref. [7].

\subsection{Computational procedures}

A recursive approach is required to give due consideration to the aerodynamic couplings among the propeller and other aerodynamic surfaces. However, a coupled analysis requires much computing time. As an alternative for an airplane with the propeller installed in front of the fore fuselage, the flow disturbance around the propeller due to the airframe can be ignored with reasonable accuracy. In such a case, the computations can be carried out in a sequential manner (Fig. 8). The induced velocities required for the computation of the air loads over the striped wing elements can be obtained using the vortex strengths determined with the 
VLM, where the induced velocity field due to the propeller can be estimated using the results of the propeller analysis. The fuselage air loads are computed using the wind tunnel database by ignoring the local flow disturbance due to the induced velocity field of the propeller and wings. In the event that this approach might cause discernible inaccuracy, the local aerodynamic loads computed only with the VLM can be used, as mentioned previously, for the dorsal and ventral fins. For completeness, the computational procedures for the propeller analysis are reproduced from the first paper in Fig. 9. The geometry and the vortex strengths of the propeller wake depend on the azimuthal location of the propeller blades. As a result, the instantaneous induced velocities

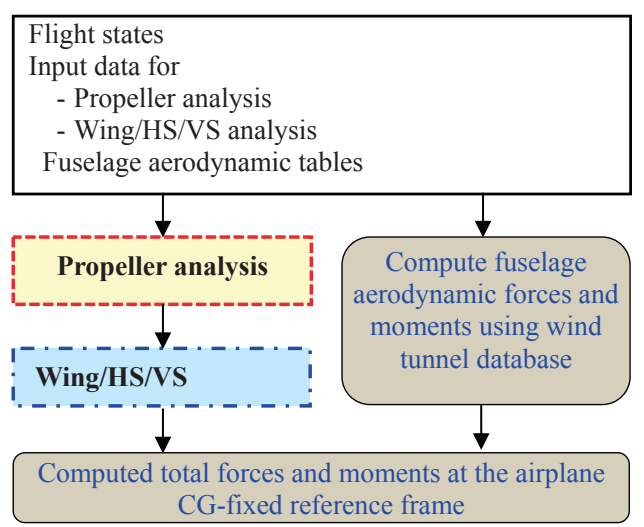

Fig. 8. Flowchart to compute total external forces and moments

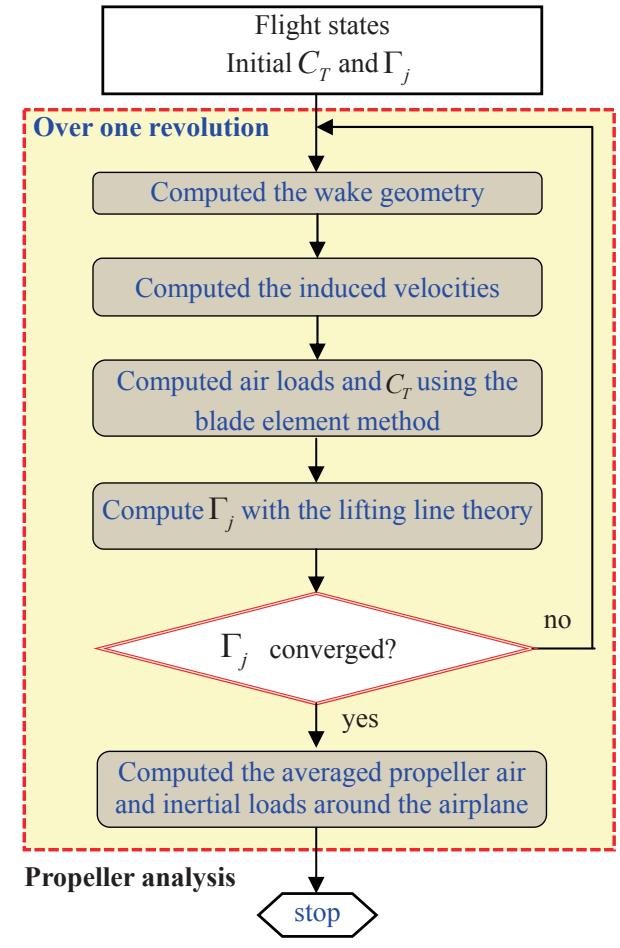

Fig. 9. Flowchart for propeller aerodynamic and inertial loads over wing surfaces also depend on these angular positions. To simplify the computation, the local induced velocities over the wing components are averaged over one propeller revolution by considering the high rotational speed of the propeller. Fig. 10 shows the flowchart to compute the aerodynamic loads of the main wing and stabilizers. The resulting sequential computational procedures guarantee reasonable computing time in predicting aerodynamic loads over wing components.

\subsection{Trade-off study of the vortex lattice geometry}

Prediction accuracy of the aerodynamic loads and the computing time are affected greatly by the VLM geometric model parameters for the propeller and the airframe. Their effects were investigated thoroughly to achieve an accurate prediction in an efficient manner. An example of such tradeoff studies is illustrated in Fig. 11, comparing the effects of the

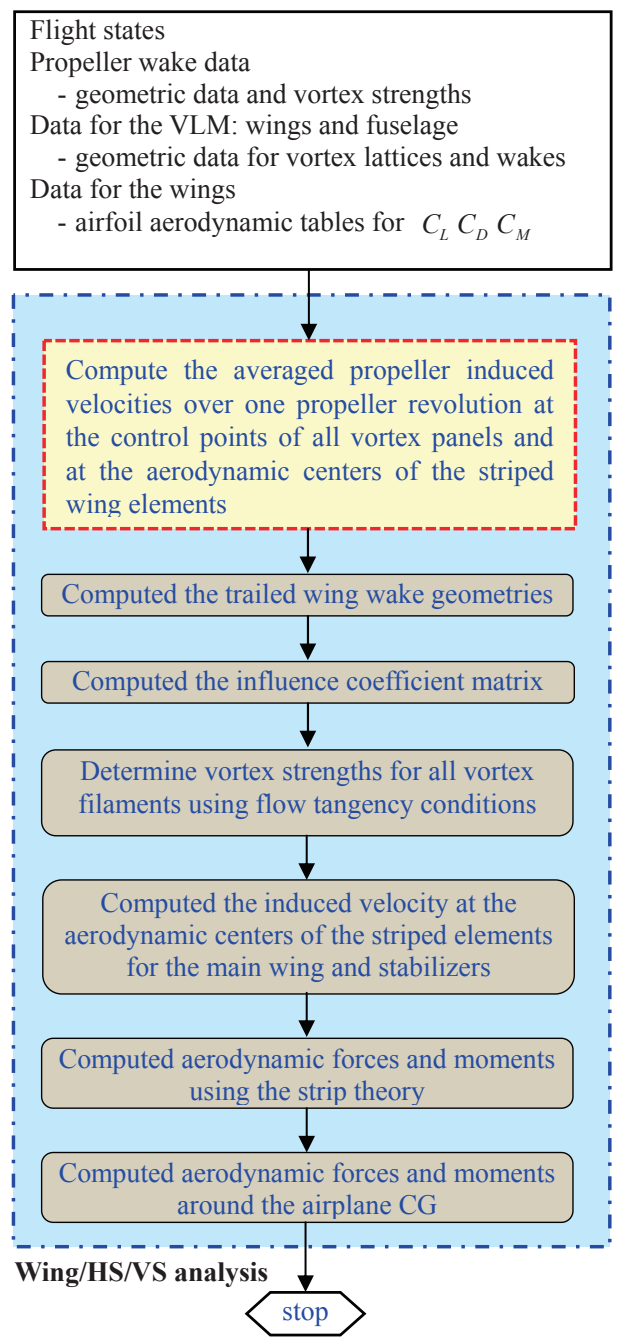

Fig. 10. Flowchart for aerodynamic models for wing components 

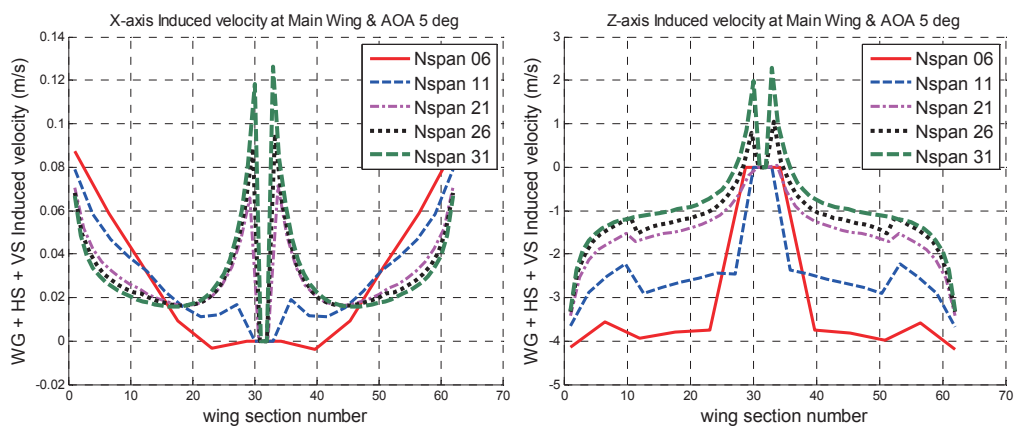

Fig. 11. Effect of the number of span-wise vortex lattices on the induced velocity

number of span-wise vortex lattices on the distribution of the velocity induced over the main wing. The results show that the induced velocities predicted with more than 31 elements of the vortex lattices have nearly the same distribution. Based on a series of trade-off studies, the geometric parameters selected for this study are presented in Table 1.

\subsection{Flight dynamics analysis modules}

The proposed aerodynamic models were integrated into the mathematical model for flight dynamics analyses, which included the trim solutions, the derivation of the linearized state equations, and the flight simulation. The trim solutions to predict steady flight characteristics require the trim kinematical relations corresponding to the steady flight conditions of interest, as shown in Ref. [8]. The trim kinematics for the general rectilinear and turning flights are implemented in the trim solution modules. The rectilinear flights can be differentiated by parameters such as the sideslip angle, the climb angle, and the flight speed, whereas, a turning flight is described by prescribing the normal load factor, the un-coordination (lateral load factor), the climb angle, the turn direction, and the flight speed. By imposing

Table 1. Geometric modeling parameters for the VLM

\begin{tabular}{|c|c|c|c|c|c|}
\hline \multicolumn{3}{|c|}{$\begin{array}{l}\text { Propeller } \\
\text { - Number of blade elements } \\
\text { - Number of azimuthal elements } \\
\text { - Wake stretching in revolutions } \\
\text { Near wake stretching }\left({ }^{\circ}\right)\end{array}$} & \multicolumn{3}{|c|}{$\begin{array}{c}21 \\
36 \\
5 \\
30\end{array}$} \\
\hline \multirow{3}{*}{$\begin{array}{l}\text { Aerodynamic surfaces } \\
\text { - spanwise lattices } \\
\text { - chordwise lattices } \\
\end{array}$} & MW & HS & VS & $\mathrm{HF}$ & VF \\
\hline & 62 & 14 & 9 & 5 & 5 \\
\hline & 7 & 3 & 4 & 21 & 21 \\
\hline \multirow{3}{*}{$\begin{array}{l}\text { Control surfaces } \\
\text { - chordwise lattices } \\
\text { - spanwise lattices }\end{array}$} & flap & aileron & \multicolumn{2}{|c|}{\begin{tabular}{|l|l} 
elevator & $r$
\end{tabular}} & rudder \\
\hline & 1 & 1 & \multicolumn{2}{|c|}{2} & 1 \\
\hline & 13 & 6 & \multicolumn{2}{|c|}{14} & 9 \\
\hline Wing wake stretching & \multicolumn{5}{|c|}{ 30x (mean wing chord) } \\
\hline $\begin{array}{l}\text { (Notation) } \\
\text { MW: main wing, } \\
\text { HS/VS: horizontal/ve } \\
\text { HF/VF: horizontal/vert }\end{array}$ & $\begin{array}{l}\text { ttical s } \\
\text { ical fus }\end{array}$ & $\begin{array}{l}\text { tbilizers } \\
\text { lage pa }\end{array}$ & & & \\
\hline
\end{tabular}

additional steady flight conditions, the trim equations can be derived in a system of non-linear algebraic equations (NAEs). These corresponding NAEs can then be solved using a quasi-Newton method to update the inverse Jacobean matrix iteratively without resorting to the time-consuming computation of the Jacobean matrix. The Martinez column update method [9] was selected for the present analyses from trade-off studies. The linearization modules were prepared to compute numerically the linear model at a given trimmed solution by applying small disturbance theory, and the reduced order models can be derived using the truncation or residualization methods [7]. The fourth-order Runge-Kutta and real-time Adams-Moulton (RTAM-3) time integrators were implemented for the flight simulation analyses.

\section{Applications}

The proposed mathematical model and analysis modules were used in the flight dynamics analyses for the KLA-100, the configuration of which is shown in Table 2 . The present methods were validated as shown in the first paper in this series [10] by comparing the simulation results with those of the flight test data gathered from the 1/5-scaled RC model of the KLA-100 in the $80 \%$ thrust condition. For completeness, the results with the $40 \%$ condition of the maximum thrust are compared in this paper. Fig. 12 shows the RC model with the two-bladed propeller and the airplane responses to the aileron doublet control are compared in Fig. 13. As mentioned in the first paper, the effects of the relative wind velocity and the misalignment in the AHRS reference frame cannot be duly considered in the measurement of attitude angles. Also, the configuration of the RC model may differ from its designed one, especially for the propeller and control surface geometries. Thus, the measured bank angle also presents discernible differences from the computed ones. However, overall roll attitude responses shows a good correlation. 
Table 2. Configuration data for KLA-100 model

\begin{tabular}{|c|c|c|c|c|}
\hline \multicolumn{5}{|c|}{ Weight, engine, and propeller } \\
\hline Max. take-off weight & \\
\hline Engine & \multirow{2}{*}{\multicolumn{4}{|c|}{100 hp Rotax 912s2 }} \\
\hline Propeller & & & & \\
\hline - Number of blades & \\
\hline - Diameter & \multirow{2}{*}{\multicolumn{4}{|c|}{$\begin{array}{l}1.726 \mathrm{~m} \\
3000\end{array}$}} \\
\hline - Maximum RPM & & & & \\
\hline \multicolumn{5}{|c|}{ Geometry of main wings (WG) and stabilizers } \\
\hline & WG & & HS & VS \\
\hline Span (m) & 9.5 & & 1.55 & 1.30 \\
\hline Chord (m) & 1.20 & & 0.65 & 1.30 \\
\hline Twist angle $\left({ }^{\circ}\right)$ & -3.0 & & 0.0 & 0.0 \\
\hline Sweep angle $\left(^{\circ}\right)$ & 0.0 & & 0.0 & 10.0 \\
\hline Dihedral angle $\left(^{\circ}\right)$ & 5.9 & & 0.0 & 0.0 \\
\hline \multicolumn{5}{|c|}{ Geometry of control surface components } \\
\hline & flap & aileron & elevator & rudder \\
\hline Chord $(\%)$ & 30 & 30 & 30 & 30 \\
\hline $\begin{array}{l}\text { Spanwise } \\
\text { Location } \\
(\mathrm{m})\end{array}$ & $\begin{array}{c}0.72 \\
\sim 3.39\end{array}$ & $\begin{array}{c}3.39 \\
\sim 4.73\end{array}$ & $\begin{array}{c}0.0 \\
\sim 1.55\end{array}$ & $\begin{array}{c}0.0 \\
\sim 1.3\end{array}$ \\
\hline
\end{tabular}

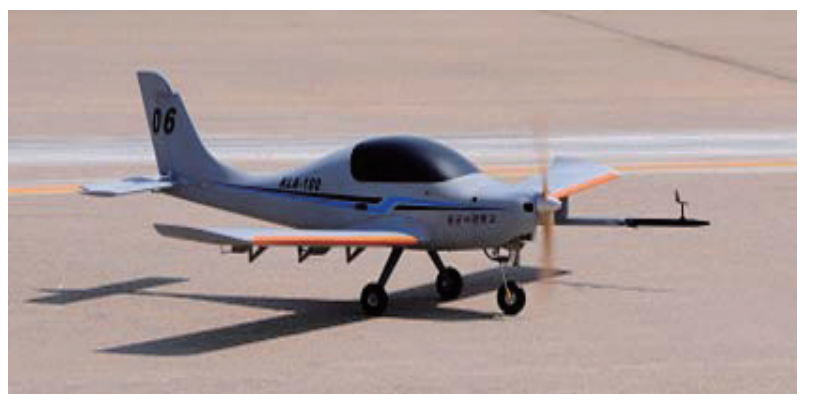

Fig. 12. Scaled RC-model of KLA-100 ready for flight tests

\section{Conclusions}

This paper focused on aerodynamic models of the fuselage, the main wing, and the stabilizers for a propellerdriven airplane and the mathematical modeling for flight dynamic analyses. Using the results from the first paper in the series, the flow field generated by the wake trailed from the propeller can be obtained over the aerodynamic surfaces of the airframe. To reflect flow disturbances due to propeller operations, in this paper, the vortex-lattice method was used to compute the induced velocity at wing components. Also, strip theory, which uses the 2D airfoil aerodynamic tables considering the viscous flow effect, was used to compute the air loads over the striped wing elements. The aerodynamic forces and moments of each wing can be obtained by integrating the contributions of each wing element. The fuselage air loads were modeled using a wind tunnel database for the airframe. For this, aerodynamic tables for the fuselage were generated by subtracting total air-loads of wing components from the airframe air loads computed using the wind tunnel test
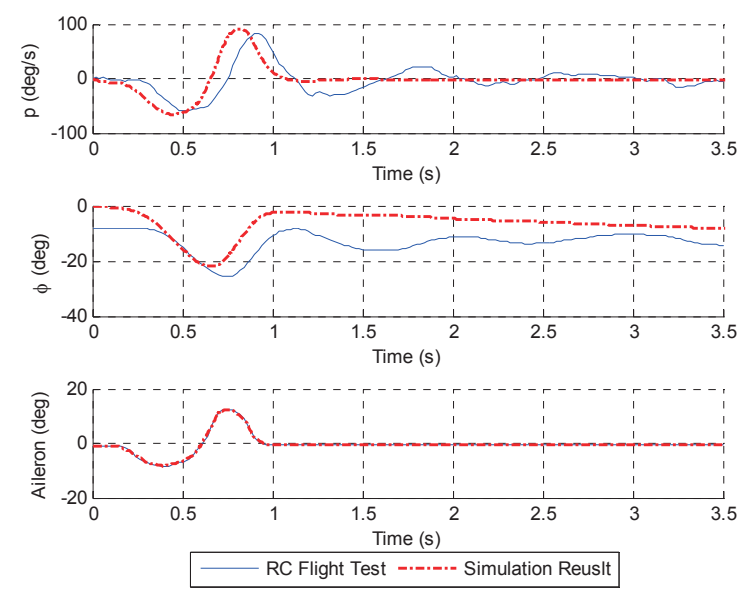

Fig. 13. Airplane responses to aileron doublet control $\left(0.4 T_{\max }\right)$

data. Thus, the static aerodynamic loads of the airframe computed with the proposed approaches are the same as those computed using the wind tunnel test data. Also, detailed procedures were described to guarantee computational efficiency in the flight dynamic analyses using the math model integrated with the propeller aerodynamic and inertial models.

The proposed methods were validated by comparing the computed control responses with flight test results for a scaled KLA-100 model. The results correlated well, indicating reasonable accuracy of the math model built in this study. Applications with the integrated math model cover three major areas of the flight dynamics analysis: the trim analyses for the general helical turn flight, linearization to achieve a linear model, and flight simulations. The trim analyses presented physically valid results over the forward speed range of interest. Thus, it can be concluded that the 

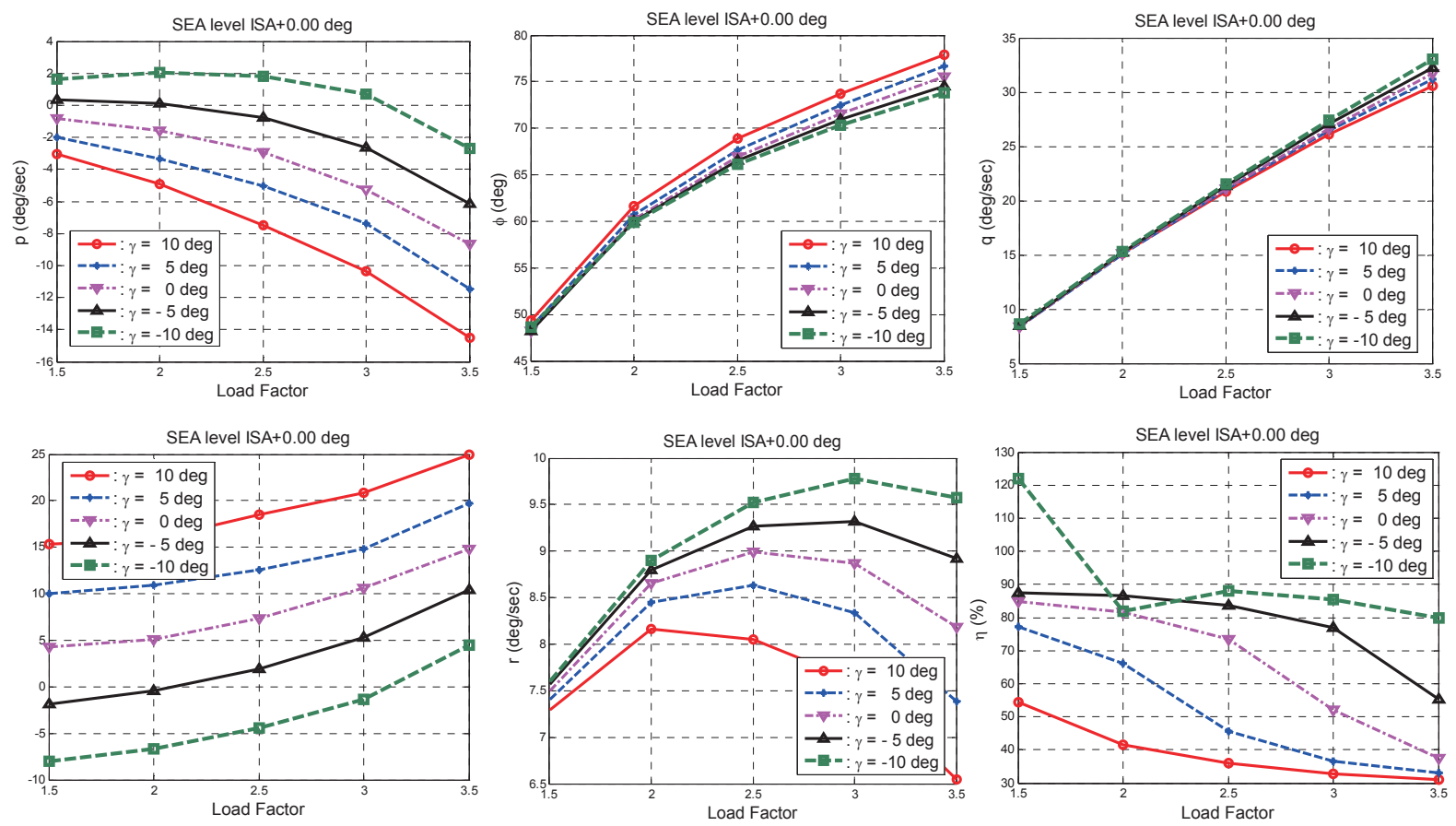

Fig. 14. Trim solutions for helical turn flights at $200 \mathrm{~km} / \mathrm{h}$ (angular rate, attitude angles, and propeller efficiency)
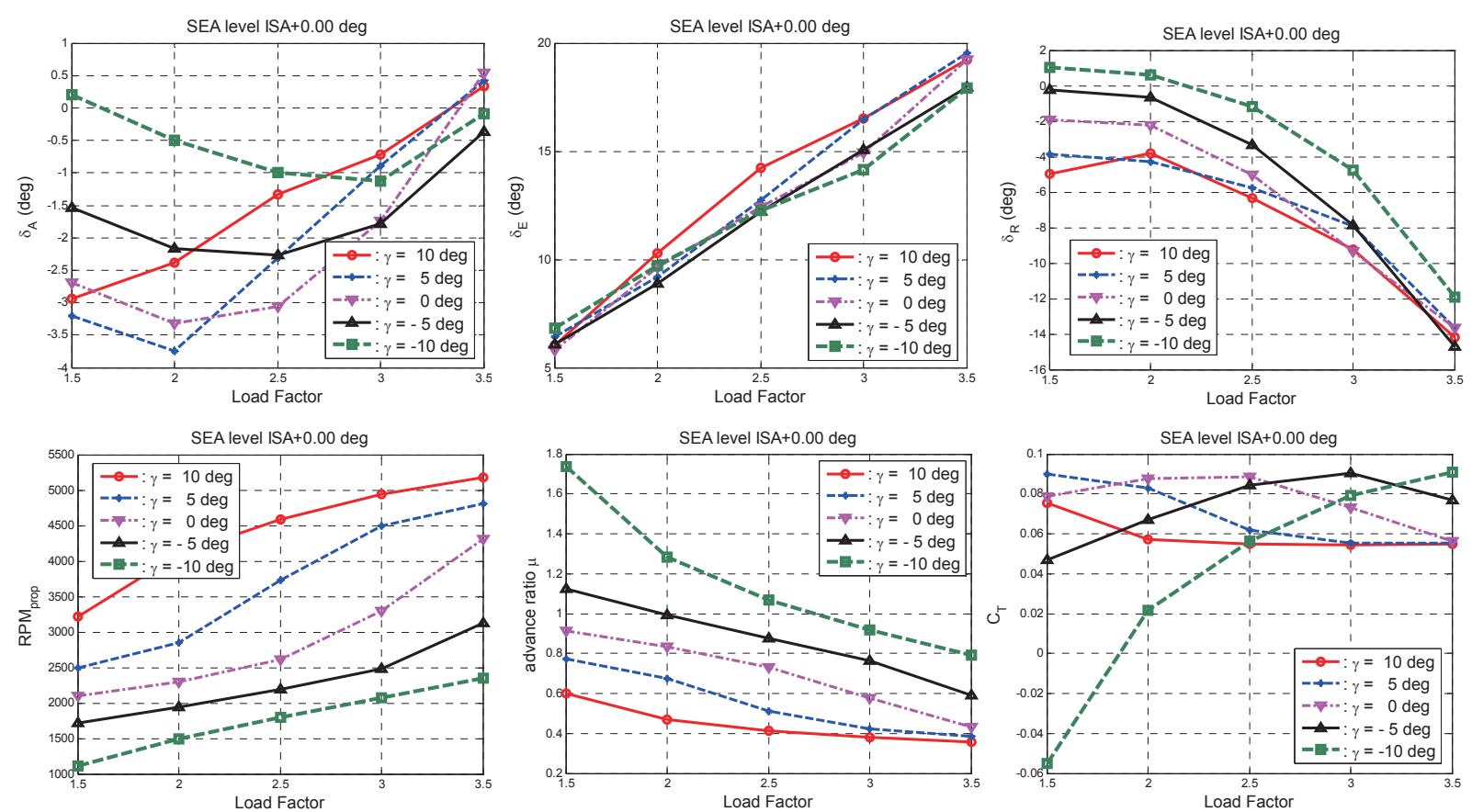

Fig. 15. Trim solutions for helical turn flights at $200 \mathrm{~km} / \mathrm{h}$ (control deflection angles and propeller operating states)

present approaches to the math model for a propeller-driven airplane are usefully applicable for flight dynamics analyses. To further improve the model fidelity, model refinement work is necessary, and will be based on various test data for the propeller airplane performance once they become available.

\section{Acknowledgments}

This research was supported by a grant (1615001723) from the Light Aircraft Development Program, funded by the Ministry of Land, Infrastructure and Transport affairs of the Korean government. 

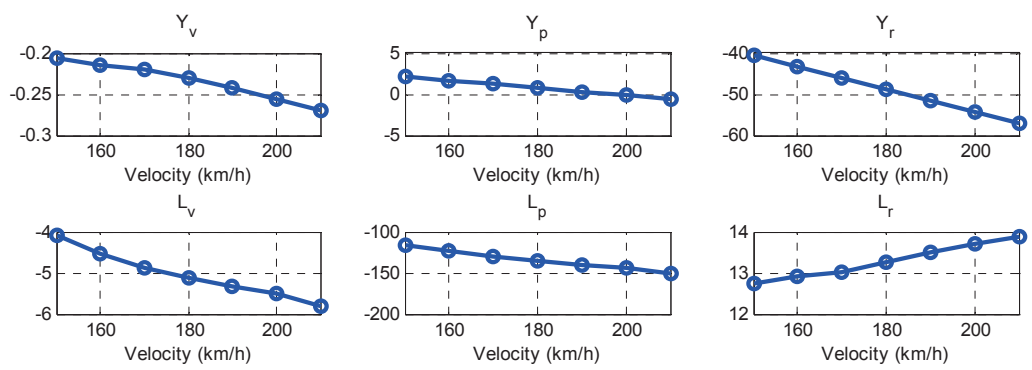

$\mathrm{N}_{\mathrm{v}}$
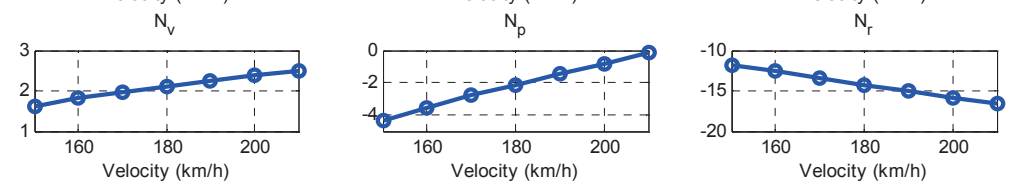

Fig. 16. Stability derivatives for the lateral-directional motion

\section{References}

[1] John D. Anderson, Jr., Fundamentals of Aerodynamics, McGraw Hill, 2011.

[2] TaeSan Park, Chang-Joo Kim, Sanh Hoon Shin, and Sunguk Hur, "Analysis Techniques of Flight Performance and Stability Analysis for Light Sports Airplane," The Korean Society for Aeronautical and Space Science, Spring conference, April, 2012.

[3] Robert F. Stengel, Flight Dynamics, Princeton University Press, 2004.

[4] J. Gordan Leishman, Principles of Helicopter Aerodynamics, Cambridge University Press, 2006.

[5] Kim C.-J., Park S. Y., Shin K. C., Kim S. H. and Jung K. H., "Study on Modelling Parameters for Rotor Free-Wake Analysis," The Korean Society for Aeronautical and Space
Science, Spring conference, December 2010.

[6] Park, S.H. and Kwon, J.H., "Implementation of Turbulence Models in an Implicit Multigrid Method," AIAA Journal, Vol.42, No.7. 2004, pp.1348 1357.

[7] David K. Schmidt, Modern Flight Dynamics, McGraw Hill, 2012.

[8] Brian L. Stevens, and Frank L. Lewis, Aircraft Control and Simulation, John Wiley \& Sons Inc., 1992.

[9] Spedicato, E., Huang, Z. and Bergamo, "Numerical Experience with Newton-like Methods for Nonlinear Algebraic Systems," Computing, Vol. 58, No. 1, 1997, pp. 69-89.

[10] Chang-Joo Kim, et. al., "Flight Dynamic Analyses of Propeller-Driven Airplane (I): Aerodynamic and Inertial Modelling of Propeller," International Journal of Aeronautical and Space Sciences, Vol. 15, No. 4, 2014, pp. 345-355. 